\title{
Statistical Description of Magnetic Domains in the Two Dimensional Ising Model
}

\author{
K. Lukierska-Walasek ${ }^{1}$, K. Topolski ${ }^{2}$ \\ Institute of Physics, University of Zielona Góra \\ ul. Z. Szafrana 4a, 65-516 Zielona Góra, Poland \\ e-mail:klukie@proton.if.uz.zgora.pl \\ Institute of Mathematics, Wroctaw University \\ Pl. Grunwaldzki 2/4, 50-384 Wroctaw, Poland \\ e-mail: topolski@math.uni.wroc.pl
}

(Received: 23 March 2010; accepted: 8 July 2010; published online: 27 September 2010)

\begin{abstract}
The Zipf-Mandelbrot power law and its connection with the inhomogeneity of the system has been used. We describe the statistical distributions of the domain masses in the Ising model near the phase transition induced by the temperature. For the large domain masses we observe the characteristic irregularities. The statistical distribution near the critical point appears to be of the Pareto type.
\end{abstract}

Key words: Ising model, phase transition, the Zipf-Mandelbrot power law

For the description of the phase transition we use the Ising model [1]. This model is one of the simplest models which is used to describe the phase transition in the ferromagnet. Apart from this, the Ising model is known due to several applications as: the percolation [2], a trading activity [3], sociophysics [4] and others. A common feature in these problems is the presence of two choices of variable $S= \pm 1$. However, all the results one can generalize to many values of $S$, for instance in Potts model.

The Hamiltonian for the simplest case of the Ising model is:

$$
H=-\frac{1}{2} \sum_{i j} J_{i j} S_{i}^{z} S_{j}^{z}
$$

with a sum over all neighbouring pairs ( $z$-th component) of spins. Usually it is assumed that the crystal lattice of the ferromagnet is regular and in each site of the lattice the spin with the value $S^{z}=-1$, or $S^{z}=-1$ is localized. Two spins $i$ and $j$ interact with each other by the energy $-J$ if both spins are parallel, and $+J$ if they are oposite to each other. Results presented hereafter are obtained by applying Monte Carlo techniques, to the two-dimensional Ising model with periodic boundary conditions. We use the FortuinKasteleyn random cluster model representation of the Ising model [5]. Clusters of the Ising model were generated using the Swenson-Wang algorithm [6]. In this algorithm, clusters of spins are created by introducing bonds between neighbouring spins of the same orientation, with probability $1-\exp \left(-\Delta E / k_{B} T\right)$, where $k_{B}$ is the Boltzmann constant and $\Delta E$ is the energy required to transform a pair of equal spins to the pair of opposite ones. To each cluster we assign at random -1 or +1 orientation and different clusters receive independent orientations.

The main goal of this paper is a statistical description of the domains in the Ising model. We consider the changes in the cluster size distribution when the system approaches the critical point of the phase transition induced by the temperature. In the case of a 2-dimensional Ising model, the critical temperature $T_{c}$, is equal to $2 J / k_{B} \ln (1+\sqrt{2})$. To simplify notation, instead of Kelvin as the temperature unit we used the dimensionless $1 / k_{B} T$ units, and with this convention the critical temperature $T_{c}$ of the 2-dimensional Ising model is equal to $2.269185 \ldots$.

At the critical temperature the distribution of the cluster size is well described by the power law distribution. This leads to the Zipf-Mandelbrot power law, $x=c k^{-1 / \mu}$, where $c$ is a constant. In our case $x$ is the domain mass described as the number of spins, which are up or down in the domain, and $k$ denotes the rank order of the domain mass $x$ (the greatest cluster has rank 1, smaller 2, and so on). 
Bouchaud [7] points out the strong correlation between the Zipf-Mandelbrot power law and the inhomogeneity of the system. The slope of the regression line describing the relation between a logarithm of the cluster mass and a logarithm of its range line is determined by $\mu$. The relation between $\mu$ and the angle of the slope is of the form $(\mu)^{-1}=-\operatorname{tg} \alpha$, and $\mu$ characterizes the inhomogeneity of the physical structure of the system. The inhomogeneity of the system means that its structures become fractal (less oval) and more hierarchical. For this reason, in our consideration we shall concentrate on the sequel of random variables $X$ which we shall call $[\mu]$-variables after Bouchaud. We say that random variable $X$ is $[\mu]$-variables if for some $\mathrm{x}_{0}>0$ and $\mu>0$

$$
P(X>x)=\left(\frac{x_{0}}{x}\right)^{\mu}, \quad x>x_{0} .
$$

The main property of $[\mu]$, variable is that all its moments $m_{q}=\left\langle x^{q}\right\rangle$, with $q \geq \mu$ are infinite. The probability distribution of the cluster size seems to be $[\mu]$-distribution and the index $\mu$ may be considered as a critical exponent.

The simple technique which helps to test the heavy tails hypothesis and estimates the tail index $\mu$ is based on the following simple observation. Assume that $X$ is a $[\mu]$-variable then $\ln P(X>x) \sim-\mu \ln x+\mu \ln x_{0}$, and plotting $\left\{(\ln x,-\ln P(X>x)), x>x_{0}\right\}$ we obtain, for large $x$, a line of slope $-\mu$. For details and a formal explanation see [8].

We study the distribution of the cluster masses. The mass of the cluster is defined as the number spins which occupy bonds in the cluster. Our simulations were performed on a two dimensional lattice with periodic boundary conditions.

The size of the system in our simulations was up to $2000 \times 2000$. All simulations were started with the random initial orientation of the spins. To equilibrate the orientations of the spins in the system we perform appropriate initial Monte Carlo steps. The number of initial Monte Carlo steps, after which the spin configuration is described by the Gibbs measure, was chosen by measuring the energy per spin and the magnetization per spin. For example, to equilibrate the system on the lattice of the linear size $L=$ 2000 we perform 10000 initial Monte Carlo steps. After the Monte Carlo system had performed an appropriate number of initial steps, we took the sample of a spins configuration and analysed the cluster configuration.

We connect the statistics of domain masses with the process of approaching the critical point. Figure 1 presents the simulation results of the system with linear size $L=$ 1000 and four different temperatures $T=2.26922,2.5$, 2.85714 and 4.0. We can see that when we start to advance from the paramagnetic phase to the critical point $\left(T \rightarrow T_{c}\right)$, then the angle between the rank axis and the line which represents dependence between the logarithm of the cluster mass and logarithm of its rank increases. We see some irregularities for the large domain masses. The estimation of index $\mu$ based on data from Fig. 1 for different temperatures is presented in Table 1 .

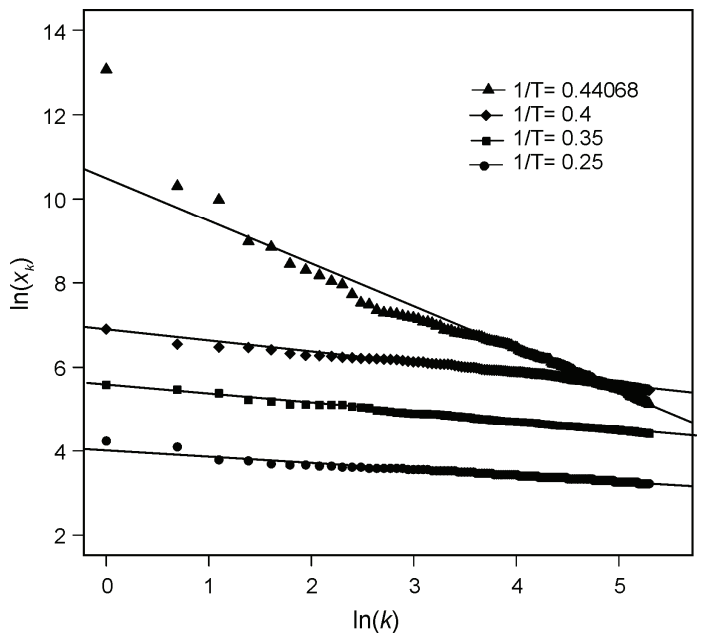

Fig. 1. Plot of logarithm of the domain masses $x$ versus logarithm of the rank index $k$ for the system with $L=1000$ and for four different temperatures $T=2.26922,2.5,2.85714$ and 4.0

Table 1. Estimation of index $\mu$ based on data for the system with linear size $L=1000$ and different temperatures $T=2.26922$, $2.5,2.85714$ and 4.0

\begin{tabular}{c|c}
\hline$T$ & $\mu$ \\
\hline 4.00000 & 6.7136 \\
\hline 2.85714 & 4.7018 \\
\hline 2.50000 & 3.8240 \\
\hline 2.26922 & 0.9854 \\
\hline
\end{tabular}

When $T$ decreases, we observe the growing domains, their structure becomes more fractal (loss of an oval) and the inhomogeneity of the system increases. For the temperature close to critical, when one domain covers almost the whole lattice and the other spin clusters are restricted to a much smaller size, we have that $\mu \approx 1$. For the temperature close to the critical temperature, $T_{c}=0.26922 \ldots$, the slope of line is close to -1 while for the higher temperatures, $T=4.0,2.85714,2.5$, the absolute value of the slopes is significantly smaller than 1 and are equal to $0.149,0.213,0.261$, respectively. 
Figure 2 represents the histogram of the domains masses at the critical point - the probability of the appearance of the cluster with mass $x$. The distribution of domain masses is $[\mu]$-distribution with $\mu \approx 1$.

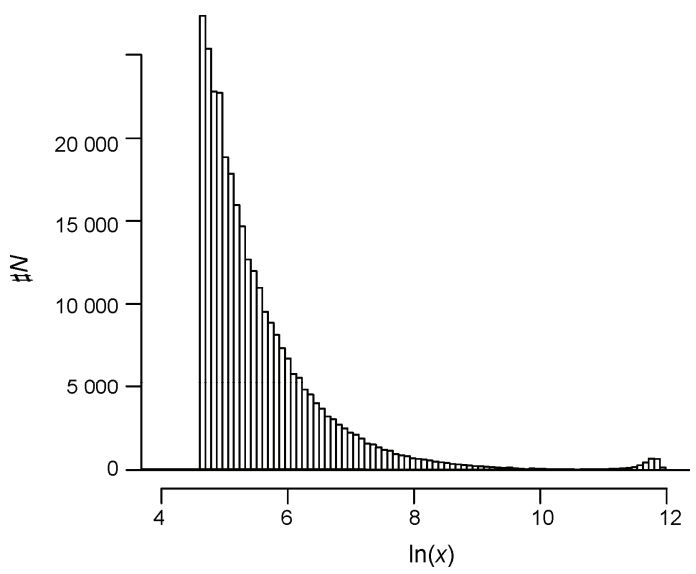

Fig. 2. Histogram of the domain masses for the system with $L=500$ and temperature $T=2.26922$ which is close to the critical temperature $T=T_{c}$

Figure 3 represents the histogram of the domain masses at temperature higher than the critical temperature $(T=$ 3.33333). In this case $\mu>1$ and the distribution of domain masses has a shorter tail than in the power law case. The histogram observations are in agreement with the percolation theory [2] and with the result of Janke and Schakel [7]. It is known in the percolation theory that $\rho_{x}$, the probability of the event, that the spin in the fixed position belongs to the domain with mass $x$, is asymptotically equal to $\rho_{x} \sim x^{-\tau} \exp (-\Theta x)$, where $\tau>0$, and $\Theta \sim\left(T-T_{p}\right)^{1 / \sigma}$, when $T \rightarrow T_{c}$. For $\tau=T_{c}$ we have $\Theta=0$ and $\rho_{x} \sim x^{-\tau}$.

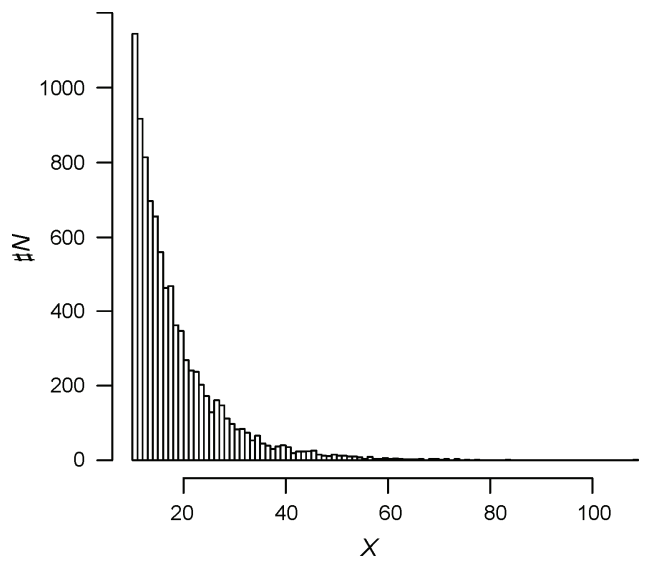

Fig. 3. Histogram of the domain masses for the system with $L=500$ and temperature $T=3.33333$
There are some experimental data for cluster size distribution. For example, the statistical description of discontinuous metal films on dielectric substrates obtained in the experiments was analyzed by Dobierzewska-Mozrzymas et al. [10], while the distributions of local field intensities in metal dielectric system were investigated by Liberman et al. [11]. Also a recent experimental test verification was performed [12] in phase ordering in a two dimensional liquid crystal using polarising microscopy.

In the paper we have shown by simulation that critical index $\mu$ from the Zipf-Mandelbrot law and the index which describes the long tail behaviour of the distribution of domains masses at the critical point are $\mu \approx 1$. At the end of the straight line representing the Zipf-Mandelbrot power law, when the phase transition appears, some characteristic irregularities are present. One can make a conjuncture that they lead to a change of statistics.

\section{References}

[1] E. Ising, Beitrag zur Theorie des Ferromagnetismus. Z. Physik 31, 253-258 (1925).

[2] D. Stauffer, Introduction to percolation theory. Taylor and Francis, London and Philadelphia (1985).

[3] R. Cont, J.P. Bouchand, Herd behavior and aggregate fluctuations in financial markets. Macroeconomics Dynamics 4, 170-196 (2000)

[4] K. Sznajd-Weron, J. Sznajd, Opinion evolution in closed community. Int. Mod. Phys. C 11, 1157-1165 (2000).

[5] C.M. Fortuin, P.W. Kasteleyn, On the random cluster model. I. Introduction and relation to other models. Physica 57, 536-564 (1972).

[6] R. Swendsen, J. Wang, Non-universal critical dynamics in Monte Carlo simulation. Phys. Rev. Lett. 58, 86-88 (1987).

[7] J.P. Bouchand, More Lévy distributions in physics. In: M.F. Shlesinger, G.M. Zasławsky, V. Frisch (ed.). Proc. Int. Workshop on Lévy Flights and Related Topics in Physics (Nice, France, 27-30 June 1994), Springer, 239-250 (1995).

[8] M. Kratz, S.I. Resnick, The qq-estimator and heavy tails. Stochastic Models 12, 699-724 (1996) and J. Beirlant, Y. Goegebeeur, J. Teugels, J. Segers, Statistics of Extremes, Theory and Applications. Wiley, Chichester, UK (2004).

[9] W. Janke, A.M.J. Schakel, Fractal structure of spin clusters and domain walls in the two-dimensional Ising model. Phys. Rev. E 71, 036703-036710 (2005).

[10] E. Dobierzewska-Mozrzymas, P. Biegański, E. Pieciul, J. Wojcik, Statistical description of systems on the basis of the Mandelbrot law: discontinuous metal films on dielectric substrates. J. Phys. Condens. Matter 11, 5561-5568 (1999).

[11] S. Liberman, F. Brouers, P. Gadenne, Levy's distributions of local fields intensities in metal-dielectric systems. Physica B 279, 56-58 (2000).

[12] A. Sicilia, J.J. Arenzon, I. Dierking, A.J. Brag, L.F. Cugliandolo, J. Martinez-Perdiguero, I. Alonso, I.C. Pintre, Experimental test of curvature-driven dynamics in the phase ordering in two dimensional liquid crystal. Phys. Rev. Lett. 101, 197801-197804 (2008). 


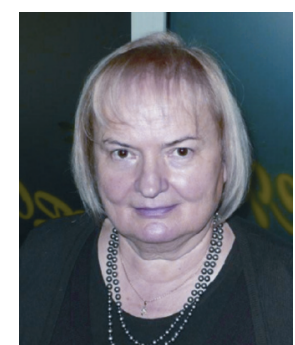

Dr Hab. Krystyna Lukierska-Walasek PhD (1975) Wrocław University, Dr hab. (1992) A.M. University of Poznań. Research: Quantum effects in the phase transitions in models: Ising model, $p$-spin model, $X-Y$ model, Potts model, spin glasses and quadrupolar glass. Methods: renormalisation group approach, replica symmetry breacking theory, cavity field method and cluster expansion method. Lately her investigations have concerned the hierarchical system statistics and the dynamics using $p$-adic numbers and computer methods.

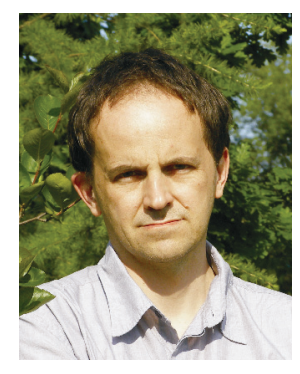

DR. KRZYSZTOF TOPOLSKI works at the Mathematical Institute of Wrocław University. His main areas of interest are the probability theory and statistics, especially application of stochastic processes to physics and biology, limit theorems and Monte Carlo simulation. He is also interested in application of the method of statistical physics to biology and social sciences. 